\title{
Cognitive Reserve Proxies Relate to Gray Matter Loss in Cognitively Healthy Elderly with Abnormal Cerebrospinal Fluid Amyloid- $\beta$ Levels
}

\author{
Eider M. Arenaza-Urquijo ${ }^{\text {a }}$, José-Luis Molinuevo ${ }^{c}$, Roser Sala-Llonch ${ }^{\mathrm{a}}$, Cristina Solé-Padullés ${ }^{\mathrm{c}}$, \\ Mircea Balasa ${ }^{\mathrm{c}}$, Beatriz Bosch ${ }^{\mathrm{c}}$, Jaume Olives ${ }^{\mathrm{c}}$, Anna Antonell ${ }^{\mathrm{c}}$, Albert Lladóc ${ }^{\mathrm{c}}$, Raquel Sánchez-Valle ${ }^{\mathrm{c}}$, \\ Lorena Rami ${ }^{\mathrm{c}, 1}$ and David Bartrés-Faz ${ }^{\mathrm{a}, \mathrm{b}, 1, *}$ \\ ${ }^{a}$ Departament de Psiquiatria i Psicobiologia Clínica, Universitat de Barcelona, Catalonia, Spain \\ ${ }^{\mathrm{b}}$ Institut d'Investigacions Biomèdiques August Pi i Sunyer (IDIBAPS), Catalonia, Spain \\ ${ }^{\mathrm{c}}$ Alzheimer's Disease and other Cognitive Disorders Unit, Hospital Clinic, Catalonia, Spain
}

Handling Associate Editor: Christine Bastin

Accepted 12 February 2013

\begin{abstract}
Cognitive reserve capacity may increase tolerance of neurodegenerative processes. However, its role regarding amyloid- $\beta\left(A \beta_{42}\right)$ deposition in cognitively normal subjects is not well understood. We aimed to investigate the association between areas showing $A \beta_{42}$-related structural changes and cognitive reserve proxies in cognitively intact subjects showing normal or abnormal $\mathrm{A} \beta_{42}$ cerebrospinal fluid (CSF) concentrations. Thirty-three subjects (aged 55-85) underwent lumbar puncture and high resolution anatomical magnetic resonance imaging analyzed by voxel-based morphometry and cortical thickness procedures. Subjects with abnormal $\mathrm{A} \beta_{42} \mathrm{CSF}$ levels showed significant left hippocampal atrophy and greater cortical thinning in parietal, temporal, and frontal regions (including the supramarginal and the anterior cingulate gyrus) compared to subjects with normal $\mathrm{A} \beta_{42} \mathrm{CSF}$ levels. Using a multivariate general linear model, we investigated the relationship between these areas and cognitive reserve proxies. We found a significant relationship between decreased volume of the left hippocampus or decreased cortical thickness of the right supramarginal gyrus and higher cognitive reserve proxies only in the group with abnormal $A \beta_{42}$ CSF levels. Thus, subjects with abnormal A $\beta_{42}$ CSF levels (which may be at a higher risk of developing Alzheimer's disease) and with high scores on cognitive reserve proxies may be tolerating a more advanced neurodegenerative process in critical cortical and subcortical regions. The present results emphasize the relevance of evaluating cognitive reserve proxies, as well as the importance of using neuroimaging techniques for early diagnosis in individuals with higher reserve.
\end{abstract}

Keywords: Aging, amyloid, cognitive reserve, hippocampus, structural MRI

\section{INTRODUCTION}

Recent research in cognitively healthy elderly individuals suggests that the pathophysiological process of

\footnotetext{
${ }^{1}$ These authors contributed equally to this study.

*Correspondence to: David Bartrés-Faz, PhD, Departament de Psiquiatria i Psicobiologia Clínica, Facultat de Medicina, Universitat de Barcelona, Casanova 143, 08036 Barcelona, Spain. Tel.: +34 9340372 64; Fax: +34 93403 52 94; E-mail: dbartres@ub.edu.
}

Alzheimer's disease (AD) begins decades before the
manifestation of clinical symptoms, that is, decades
before the diagnosis of clinical dementia. The most
commonly accepted model of AD proposes amyloid- $\beta$
$\left(\mathrm{A} \beta_{42}\right)$ peptide accumulation as a key early event in the
pathophysiological process of AD [1-3]. This means
that older individuals may harbor pathological A $\beta_{42}$
deposition without presenting any clinical symptoms.
These asymptomatic individuals may in part represent 
the preclinical phase of $\mathrm{AD}$. Interestingly, thanks to previous autopsy [4-8] and 'in vivo' $\mathrm{A} \beta_{42}$ imaging and cerebrospinal fluid (CSF) studies [9-14], we know that around one-third of these individuals with pathological $A \beta_{42}$ deposition may not become symptomatic before death. Differences in the lag phase between $A \beta_{42}$ deposition and the appearance of symptoms may be partially caused by interindividual differences in brain reserve [15] and/or cognitive reserve [16]. Brain reserve refers to interindividual differences in the neural substrate: for example, greater synaptic density or a larger number of neurons to support normal function despite pathological processes. Cognitive reserve is the capacity of the adult brain to cope with more advanced pathology by taking advantage of functional resources (for example, the capacity to use alternative networks or cognitive strategies to cope with the effects of the pathology). Common indexes reflecting brain reserve include increased intracranial or brain volumes while, as operationalized in the present article, high scores on proxy variables such as education, occupational status, and lifetime exposure to cognitively stimulating activities reflect cognitive reserve [17].

Recent Pittsburgh Compound B (PiB) and autopsy studies in healthy elderly suggested greater brain reserve in terms of larger temporal and hippocampal areas in cognitively spared individuals with high $A \beta_{42}$ deposition $[18,19]$. Furthermore, several amyloid imaging and CSF studies suggested that cognitive reserve may have a modulating role at different levels by: i) influencing the relationship between $A \beta_{42}$ accumulation and cognition [20-22]; ii) acting directly upon $A \beta_{42}$ accumulation, that is, slowing $A \beta_{42}$ deposition [23]; or iii) permitting longer tolerance of $A \beta_{42}$-related neural death or whole brain atrophy [9]. This last point is of special interest since previous cognitive reserve studies in $\mathrm{AD}$ showed that higher cognitive reserve proxies allow individuals to cope with greater AD-related cortical thinning [24]. Inverse associations between cognitive reserve proxies and brain structure or metabolism have frequently been described in the multimodal cortex, for example in frontal and temporoparietal areas in healthy elderly and in patients with AD [25]. Interestingly, the spatial pattern of $A \beta_{42}$ deposition [26] and $A \beta_{42-}$ related atrophy (e.g., [27]) overlap notably with areas of the multimodal cortex. If cognitive reserve allows individuals with preclinical AD to tolerate greater atrophy, especially in $A \beta_{42}$-related areas, this would have implications for early diagnosis. However, the role of cognitive reserve proxies in modulating gray matter atrophy or cortical thinning is not well understood, since both reductions [28, 29] and increases [30, 31] in gray matter volume and cortical thickness as a function of cognitive reserve proxies have been reported in previous studies in healthy elderly. These contradictory findings may be related to the inclusion of subjects at a higher risk of developing dementia (for example, presenting high $\mathrm{A} \beta_{42}$ deposition), and thus, partially representing preclinical $\mathrm{AD}$, in the majority of neuroimaging studies. In this study we aimed first to identify areas of $A \beta_{42}$-related cortical thinning and gray matter loss by comparing elderly with normal and abnormal $A \beta_{42}$ CSF levels. Second, we aimed to investigate whether there were differences in the relationships between cognitive reserve proxies and $A \beta_{42}$-related gray matter atrophy in the two groups. In agreement with the robust inverse associations between cognitive reserve proxies and measures of brain integrity observed in $\mathrm{AD}$, we hypothesized that the associations would be reversed in the two groups (i.e., positive in the group with normal $\mathrm{A} \beta_{42} \mathrm{CSF}$ levels, negative in the group with abnormal $\mathrm{A} \beta_{42} \mathrm{CSF}$ levels).

\section{MATERIALS AND METHODS}

\section{Participants}

Thirty-three volunteers were recruited from the Alzheimer's disease and other Cognitive Disorders Unit, Neurology Service, at Hospital Clínic, Barcelona, Spain. The study was approved by the local ethics committee and all subjects gave informed written consent. None of the participants selected had a medical history of acute neurologic deficit compatible with transient ischemic attack or stroke, or radiologic evidence of stroke. Subjects did not meet criteria for dementia or mild cognitive impairment (MCI), and their cognitive performance was not less than $-1.5 \mathrm{SD}$ on any test included in the neuropsychological examination [32]. Mini-Mental State Examination (MMSE) scores were between 24 and 30. On the Global Deterioration Scale (GDS) rating [33], all subjects presented normal functional capacity (GDS ratings 1 or 2).

\section{CSF analyses and group classification}

Ten milliliters of CSF were obtained during the morning (9:00 to 12:00 a.m) and samples were centrifuged and stored in polypropylene tubes at $80^{\circ} \mathrm{C}$. The $\mathrm{A} \beta_{1-42}$ at threonine 181 in CSF was measured by enzyme-linked immunosorbent assay (Innogenetics, Ghent, Belgium). Patients were not fasted. As patients 
Table 1

Demographical variables (including cognitive reserve proxies and years of education), global cognitive function (Mini-Mental State Examination, MMSE), cerebrospinal fluid (CSF) characteristics, and $A P O E$ status of the subjects included in the study. P-tau: phosphorylated tau

\begin{tabular}{lccr}
\hline & Normal $\mathrm{A} \beta_{42}$ CSF levels mean $(\mathrm{SD})$ & Abnormal $\mathrm{A}_{42}$ CSF levels mean $(\mathrm{SD})$ & $T$-test/Chi square $(p$ value $)$ \\
\hline$n$ & 16 & 17 & - \\
Male: Female & $6: 10$ & $8: 9$ & $0.30(0.57)$ \\
Age & $68.06(5.66)$ & $72.41(7.83)$ & $-1.81(0.07)$ \\
Cognitive reserve proxy & $13.60(5.08)$ & $12.38(4.81)$ & $0.84(0.40)$ \\
Years of education & $10.31(4.95)$ & $9.52(4.72)$ & $0.46(0.64)$ \\
MMSE & $28.31(1.14)$ & $28.05(1.48)$ & $0.55(0.58)$ \\
APOE $\varepsilon 4$ carriers $(\%)$ & $3(18.8 \%)$ & $6(35.3 \%)$ & $1.13(0.28)$ \\
CSF A $\beta_{42}(\mathrm{pg} / \mathrm{ml})$ & $688.72(129.93)$ & $372.80(101.60)$ & $7.80(0.001)$ \\
CSF p-tau $(\mathrm{pg} / \mathrm{ml})$ & $57.81(21.19)$ & $73.03(45.83)$ & $13.21(0.23)$ \\
\hline
\end{tabular}

were participants in a quality control program, determinations in our laboratory of $A \beta_{42}$ at threonine 181 values were within mean 2 SD. A cutoff of $500 \mathrm{pg} / \mathrm{mL}$ [34] was used to dichotomize the sample into 16 subjects with abnormal $A \beta_{42}$ CSF levels $(<500 \mathrm{pg} / \mathrm{mL})$ and 17 with normal $\mathrm{A} \beta_{42}$ CSF levels $(>500 \mathrm{pg} / \mathrm{mL})$ (see Table 1). The percentage of subjects showing abnormal $A \beta_{42}$ CSF levels was higher than might be expected in the population $[8,11]$.

\section{Individual cognitive reserve estimations}

All but two subjects included in the study underwent cognitive reserve proxy assessment. Proxies of cognitive reserve were estimated using a customized questionnaire [35] focusing on years of education and lifespan cognitive activities. Information regarding years of education was quantified and ranged from "no formal education" to "university education". In detail, they were coded as $0=$ no formal education, $1=$ self-taught reader and writer, $2=$ primary school, $3=$ secondary school, and $4=$ higher or university education. Occupational information was coded as follows: $0=$ unskilled, $1=$ skilled manual, $2=$ skilled non-manual or technician, $3=$ professional (university degree), and $4=$ manager or director (university degree). We also recorded information about musical studies, languages, and lifetime involvement in leisure activities such as reading and playing intellectual games and their frequency. The questionnaire has a maximum score of 25 , with 25 representing the highest cognitive reserve.

\section{Neuropsychological and functional assessment}

Participants were administered a one-hour neuropsychological battery test by a trained neuropsychologist. The battery included memory, language, praxis, visual perception, and frontal functions assessment. All neuropsychological scores were adjusted for age and educational level. Normative neuropsychological data and the neuropsychological battery have been described in detail elsewhere [32].

\section{MRI acquisition and processing}

High resolution 3D structural dataset (T1-weighted MP-RAGE, TR $=2300 \mathrm{~ms}, \mathrm{TE}=2.98 \mathrm{~ms}, 240$ slices, FOV $=256 \mathrm{~mm}$, matrix size $=256 \times 256$; Slice thickness $=1 \mathrm{~mm}$ ) were acquired on a $3 \mathrm{~T}$ MRI scanner (Magnetom Trio, Tim, Siemens Medical Systems, Germany) for the 33 subjects.

Cortical reconstruction of the structural images was performed with the FreeSurfer image analysis suite, version 4.3.1. (http://surfer.nmr.mgh.harvard.edu/). The procedures carried out with FreeSurfer software include removal of non-brain data, intensity normalization [36], tessellation of the gray matter/white matter boundary, automated topology correction [37, 38 , and accurate surface deformation to identify tissue border [39, 40]. Reconstructed and registered individual cortical thickness maps were smoothed using a Gaussian Kernel of $15 \mathrm{~mm}$ Full-Width Half Maximum (FWHM). Structural MRI data were also processed with FSL-VBM, a voxel-based morphometry style analysis included in FSL suite [41] (http://www.fmrib.ox.ac.uk/fsl). First, brain extraction on all T1 images was carried out [42]. All brainextracted images were segmented into gray matter, white matter, and CSF [43]. Gray matter images were then registered to the gray matter ICBM-152 template. The registered gray matter images were concatenated into a 4D image and averaged to create the studyspecific gray matter template based on a non-linear registration. All the gray matter images were nonlinearly registered to the study-specific template and concatenated into a 4D image introducing a compensation or modulation for the non-linear component of the transformation (Jacobian of the warp field). Finally 
Table 2

Neuropsychological performance of the two groups included in the study. Between group statistical differences are reported. FCSRT: Free and cued selective reminding test; ROCF: Rey-Osterrieth Complex Figure; CERAD battery: Consortium to Establish a Registry of Alzheimer's Disease; VOSP: Visual Object and Space Perception battery; WAIS: Weschler Adult Intelligence Scale; TMT: Trail Making Test

\begin{tabular}{|c|c|c|c|}
\hline & Normal $A \beta_{42}$ CSF levels mean (SD) & Abnormal $\mathrm{A} \beta_{42}$ CSF levels mean (SD) & $T$-test ( $p$ value) \\
\hline FCSRT: Learning & $25.38(5.23)$ & $21.06(7.53)$ & $1.88(0.07)$ \\
\hline FCSRT: Total learning & $41.00(7.27)$ & $39.94(6.67)$ & $0.43(0.67)$ \\
\hline FCSRT: Recall & $10.00(1.51)$ & $8.19(3.56)$ & $1.87(0.07)$ \\
\hline FCSRT: Total recall & $14.25(1.23)$ & $13.63(2.50)$ & $0.89(0.38)$ \\
\hline ROCF: Immediate recall & $17.87(4.12)$ & $14.97(5.97)$ & $1.56(0.13)$ \\
\hline ROCF: Delayed recall & $18.25(4.05)$ & $14.88(6.45)$ & $1.73(0.09)$ \\
\hline Boston naming test & $54.62(4.54)$ & $53.50(4.54)$ & $0.55(0.59)$ \\
\hline Semantic fluency (Animals) & $18.06(4.84)$ & $18.18(4.38)$ & $-0.07(0.94)$ \\
\hline Phonologic fluency $(\mathrm{F}, \mathrm{A}, \mathrm{S})$ & $32.13(15.33)$ & $29.06(11.44)$ & $0.65(0.52)$ \\
\hline CERAD: Praxis & $10.25(1.34)$ & $10.41(.87)$ & $-0.41(0.68)$ \\
\hline VOSP : Number location & $9.06(1.12)$ & $8.69(1.74)$ & $0.72(0.48)$ \\
\hline VOSP: Incomplete letters & $19.44(.96)$ & $19.19(1.76)$ & $0.49(0.62)$ \\
\hline Digit (WAIS III): Direct & $8.08(1.66)$ & $8.21(1.97)$ & $-0.19(0.85)$ \\
\hline Digit (WAIS III): Inverse & $5.08(1.55)$ & $5.21(1.63)$ & $-0.22(0.82)$ \\
\hline Digit (WAIS III): Total & $13(2.97)$ & $13.13(2.72)$ & $-0.12(0.90)$ \\
\hline TMT A & $56.67(17.21)$ & $50.82(17.05)$ & $0.39(0.69)$ \\
\hline TMT B & $160.25(82.01)$ & $154.35(76.54)$ & $0.21(0.83)$ \\
\hline
\end{tabular}

they were smoothed by a range of Gaussian kernels $($ sigma $=2,3,4 \mathrm{~mm})$. The concatenated processed data, smoothed at 3 sigma (approximately $7 \mathrm{~mm}$ FWHM), was used to run randomize.

\section{Statistical analyses}

\section{Demographical and neuropsychological variables}

Student's $t$-test or chi square was performed with Statistical Package for the Social Sciences (SPSS) for Windows (V.17.0). Results were considered as significant at $p<0.05$.

\section{Cortical thickness and VBM analyses: \\ Between-group comparison}

Statistical analysis of differences in cortical thickness maps (elderly with abnormal versus elderly with normal $\mathrm{A} \beta_{42} \mathrm{CSF}$ ) were tested in both hemispheres using vertex-wise $t$-test as implemented in QDEC from FreeSurfer. We used a standard general linear model (GLM) design and corrected for Family-Wise Error (FWE) using a Monte-Carlo permutation-based testing (10000 permutations). For the voxel-by-voxel between-group comparison (subjects with normal/abnormal $\mathrm{A} \beta_{42} \mathrm{CSF}$ ), a standard GLM design (permutations $=5000$ ), and threshold-free cluster enhancement correction was used, controlling FWE rate. In order to isolate the role of $A \beta_{42}$ levels on brain structure, we included demographical variables (age and gender), $A P O E$ status (categorized as $A P O E / 4$-allele carrier/non-carrier, see results) and CSF p-tau levels $[44,45]$ as covariates.

\section{Group by cognitive reserve proxy interaction analyses}

Volume and cortical thickness of the significant clusters showing between-group differences (subjects with abnormal< subjects with normal $\mathrm{A} \beta_{42} \mathrm{CSF}$, see results) were extracted. We carried out a multivariate GLM as implemented in SPSS. Cortical thickness and gray matter volume of the significant clusters were introduced as dependent variables, group as categorical predictor of the model (fixed factor), and cognitive reserve proxies as independent variable. We investigated the interactive effect of group and cognitive reserve proxies on left hippocampal volume and right supramarginal, left caudal cingulate, left superior temporal, and left frontal parsopercularis cortical thickness. For the statistically significant results, multiple regression coefficients were calculated separately in each group. Results were considered as significant at $p<0.05$.

\section{RESULTS}

Demographical variables and neuropsychological assessment

The two groups did not differ in any of the demographical variables studied (see Table 1 for details) or cognitive function (see Table 2). However, the between-group comparison showed a trend toward significance $(p=0.07)$ for learning and delayed recall memory performances. 
Differences in cortical thickness and gray matter volumes (Elderly with Abnormal $<$ Normal $A \beta_{42}$ CSF Levels)

Statistically significant cortical thickness reductions were observed in subjects with abnormal $A \beta_{42} \mathrm{CSF}$ levels than in those with normal $A \beta_{42}$ CSF levels at $p<0.05$ FWE corrected. The anatomical pattern included superior and middle temporal areas, frontal areas (lateral orbitrofrontal, parsopercularis, parstriangularis, rostral middle frontal, caudal middle frontal, superior frontal, and caudal cingulate) in the left hemisphere, and parietal regions including the supramarginal gyrus in the right hemisphere (see Fig. 1A, Table 3). Moreover, the voxel-based statistical analyses for the group comparison revealed gray matter differences in the left hippocampus. Elderly with abnormal $\mathrm{A} \beta_{42} \mathrm{CSF}$ levels showed lower hippocampal volume than those with normal $A \beta_{42}$ CSF levels (see Fig. 1B, Table 3). When a less strict correction was used for the VBM analysis (uncorrected $p<0.001$ ), the pattern of atrophy was extended to temporal and frontal areas (data not shown). No results were found when the opposite contrast (subjects with abnormal $>$ subjects with normal $A \beta_{42}$ CSF levels) was tested.

\section{Relationships between cognitive reserve proxies, volume, and cortical thickness}

We observed a statistically significant groupby-cognitive reserve proxy interaction in the left hippocampal volume $(\mathrm{F}=6.19 ; p=0.02)$ and the right supramarginal gyrus $(\mathrm{F}=7.17 ; p=0.01)$ (Fig. 2). Correlation coefficients showed a significant negative association between left hippocampal volume and cognitive reserve proxies (see Fig. 2, in red, $r=-0.554$; $p=0.03$ ) and between the cortical thickness of the right supramarginal gyrus and cognitive reserve proxies (see Fig. 2, in red, $r=0-0.516 ; p=0.04$ ) in elderly with abnormal $A \beta_{42}$ CSF levels. This association was not significant (hippocampal volume: $r=0.17, p=0.52$; supramarginal cortical thickness: $r=0.38, p=0.16$ ), in elderly with normal $A \beta_{42}$ CSF levels.

\section{DISCUSSION}

In the present study, we aimed to investigate the differences in the relationship between areas showing $\mathrm{A} \beta_{42}$-related structural changes and cognitive reserve proxies in healthy elderly subjects showing biomarker positivity for CSF $A \beta_{42}$ levels (at higher risk of developing $\mathrm{AD}$ ) compared to elderly with normal $A \beta_{42}$ levels. We first identified regions showing differences in regional cortical thinning and gray matter atrophy between groups. Elderly with abnormal $\mathrm{A} \beta_{42} \mathrm{CSF}$ levels showed greater cortical thinning in temporal, parietal, and frontal regions and greater left hippocampal atrophy than elderly with normal $\mathrm{A} \beta_{42} \mathrm{CSF}$ levels. Higher cognitive reserve proxies were related to greater hippocampal atrophy and cortical thinning of the supramarginal gyrus only among subjects with abnormal $A \beta_{42}$ CSF levels, suggesting that among these individuals those with higher cognitive reserve proxies are tolerating greater $A \beta_{42}$-related atrophy and cortical thinning.

Our results showing greater cortical thinning and regional atrophy in elderly with abnormal $A \beta_{42}$ CSF levels corroborate previous studies demonstrating structural changes in these individuals. The pattern of thinning that we found (i.e., superior temporal, supramarginal gyrus, anterior cingulate, and inferior frontal) shows differences as well as similarities with regard to previous studies investigating gray matter atrophy related to $A \beta_{42}$ deposition as measured by PET imaging or CSF levels. Dickerson et al. [27] demonstrated a signature of AD in cortical anatomy by comparing healthy elderly individuals to mild $\mathrm{AD}$ subjects. This cortical signature of mild AD included the temporal and parietal cortex (including angular and supramarginal gyrus and precuneus) and the inferior frontal gyrus. In the same study, these areas were defined as regions of interest (ROI) and investigated in a small sample of cognitively healthy elderly with high brain amyloid binding (as detected by PiB-PET imaging). Only the temporal pole and, as we reported in the present study, the inferior frontal cortex showed significant cortical thinning compared to subjects with low brain amyloid binding. However, Dickerson et al. [27] found a pattern of subtle thinning in all the regions, including areas described in the present study such as the supramarginal gyrus. Anterior cingulate and superior temporal lobe were not regions described in Dickerson et al.'s proposed AD signature; however, cortical thinning of these areas has been reported in previous studies comparing cognitively normal individuals with high and low $A \beta_{42}$ deposition [46-48]. The discrepancies in the $A \beta_{42}$-related cortical thinning in cognitively healthy elderly described in the different studies are most likely the result of methodological differences. Unlike in the present study, ROI-based approaches [27, 46, 48] are mainly used, although a combination of ROI-based and whole brain approaches has also been applied [47] to study elderly with abnormal $\mathrm{A} \beta_{42}$ CSF levels. Measures of amyloid 
A Left hemisphere
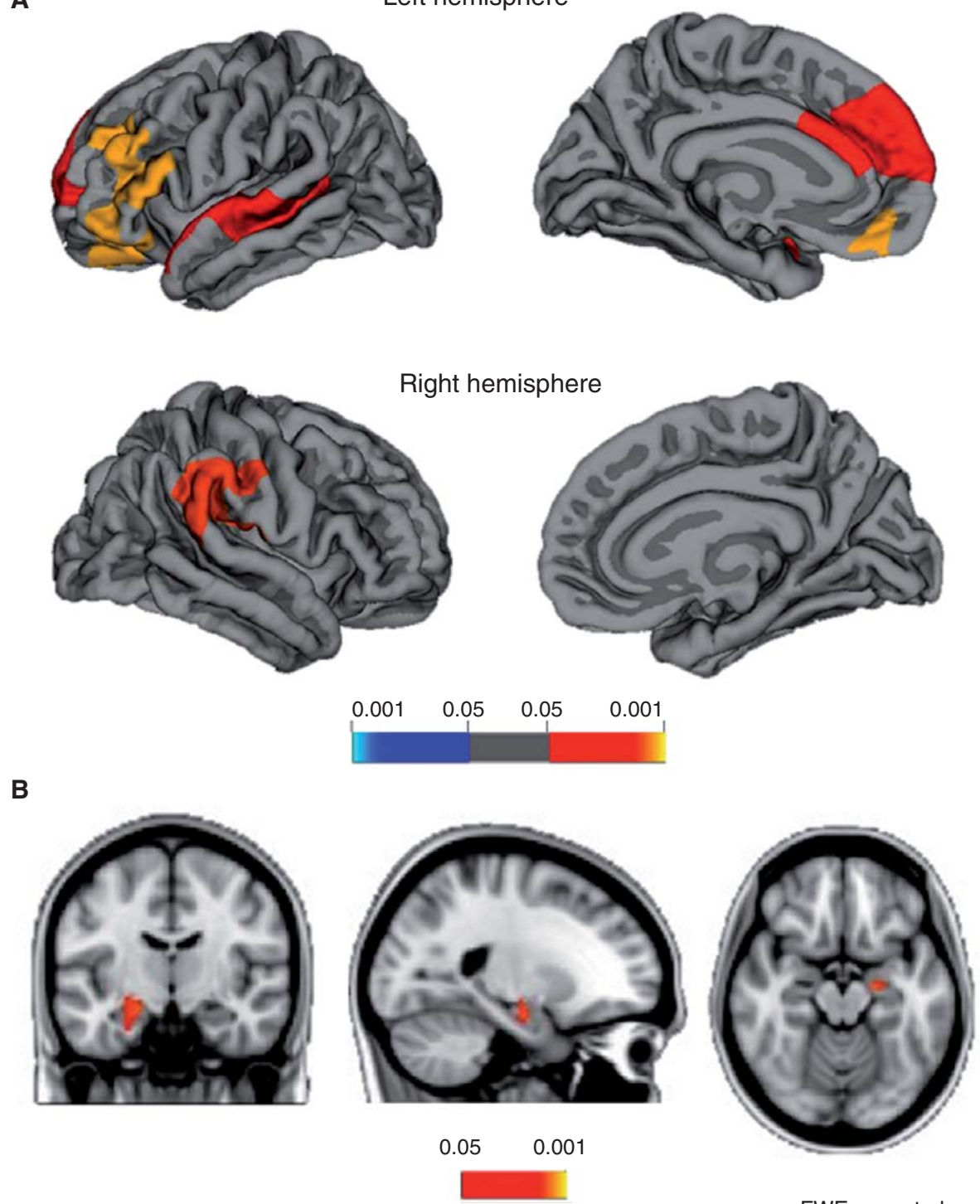

FWE corrected

Fig. 1. Results of cortical thickness (A) and voxel-based morphometry analyses (B) showing differences between healthy elderly subjects with normal versus abnormal $\mathrm{A} \beta_{42}$ CSF levels (abnormal < normal $\mathrm{A} \beta_{42}$ CSF levels). All results are FWE-corrected.

Table 3

Cortical thickness and voxel-based morphometry (VBM) results for between-group comparison. Reported clusters are corrected for multiple comparisons (FWE). The coordinates $\mathrm{x}, \mathrm{y}$ and $\mathrm{z}$ refer to the anatomical location, indicating Tailairach standard stereotactic space. L: left, R. right

\begin{tabular}{lccccccc}
\hline $\begin{array}{l}\text { Cortical area/ } \\
\text { Brain region }\end{array}$ & $\begin{array}{c}\text { Cluster size } \\
\left(\mathrm{mm}^{2}\right)\end{array}$ & \multicolumn{3}{c}{$\begin{array}{c}\text { Coordinates } \\
x y z\end{array}$} & $\begin{array}{c}\text { Normal A } \beta_{42} \\
\text { CSF levels mean (SD) }\end{array}$ & $\begin{array}{c}\text { Abnormal A } \beta_{42} \\
\text { CSF levels mean (SD) }\end{array}$ & $p$ value \\
\hline Cortical thickness & & & & & & & \\
L Superior temporal & 2580.30 & -51.9 & -15.6 & -6.7 & $2.72(0.12)$ & $2.50(0.10)$ & 0.009 \\
L Frontal parsopercularis & 4480.98 & -47.4 & 25.2 & 10.1 & $2.60(0.16)$ & $2.41(0.10)$ & 0.008 \\
L Caudal cingulate & 2661.77 & -1 & 18.3 & 17.7 & $2.84(0.19)$ & $2.65(0.16)$ & 0.008 \\
R Supramarginal & 2943.54 & 43.5 & -32.7 & 24.7 & $2.56(0.11)$ & $2.34(0.20)$ & 0.002 \\
$V B M$ & & & & & & & \\
L Hippocampus & 73 & -22 & -14 & -18 & $0.63(0.04)$ & $0.53(0.05)$ & 0.035 \\
\hline
\end{tabular}



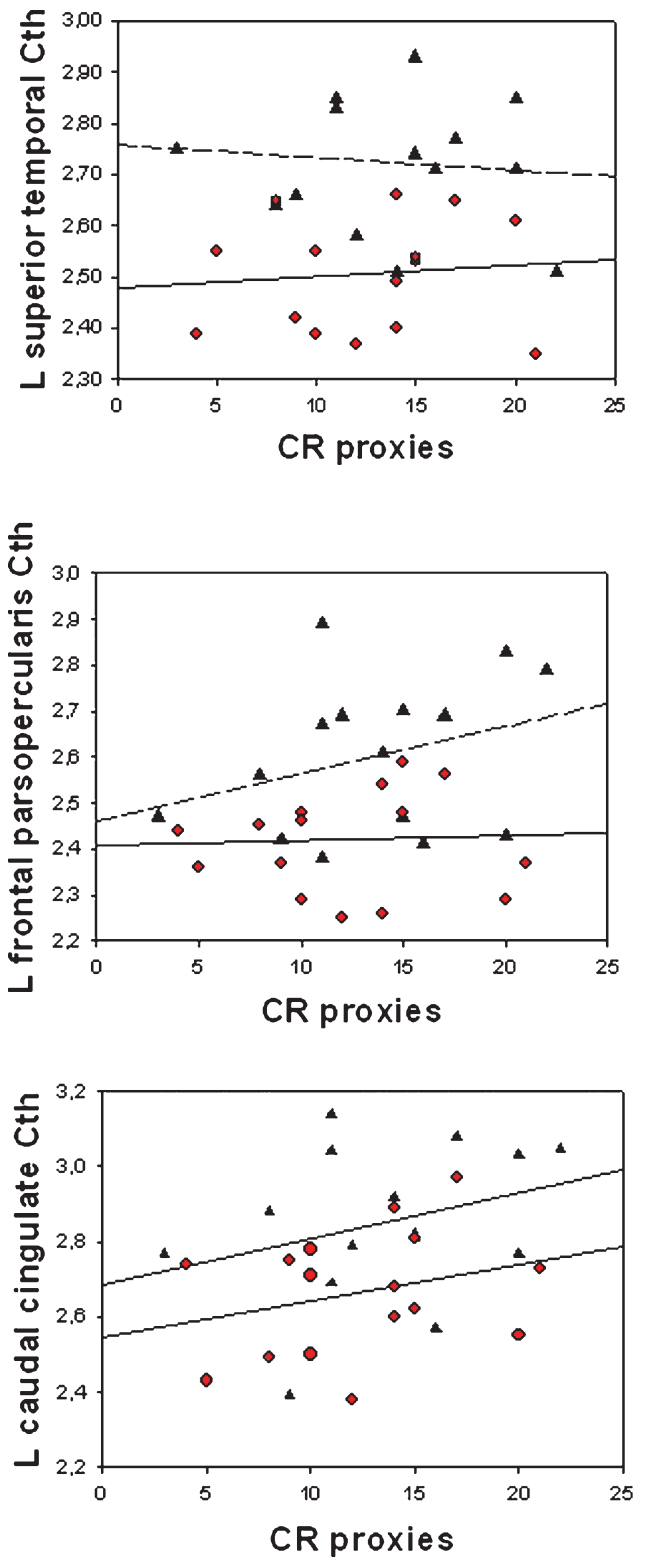
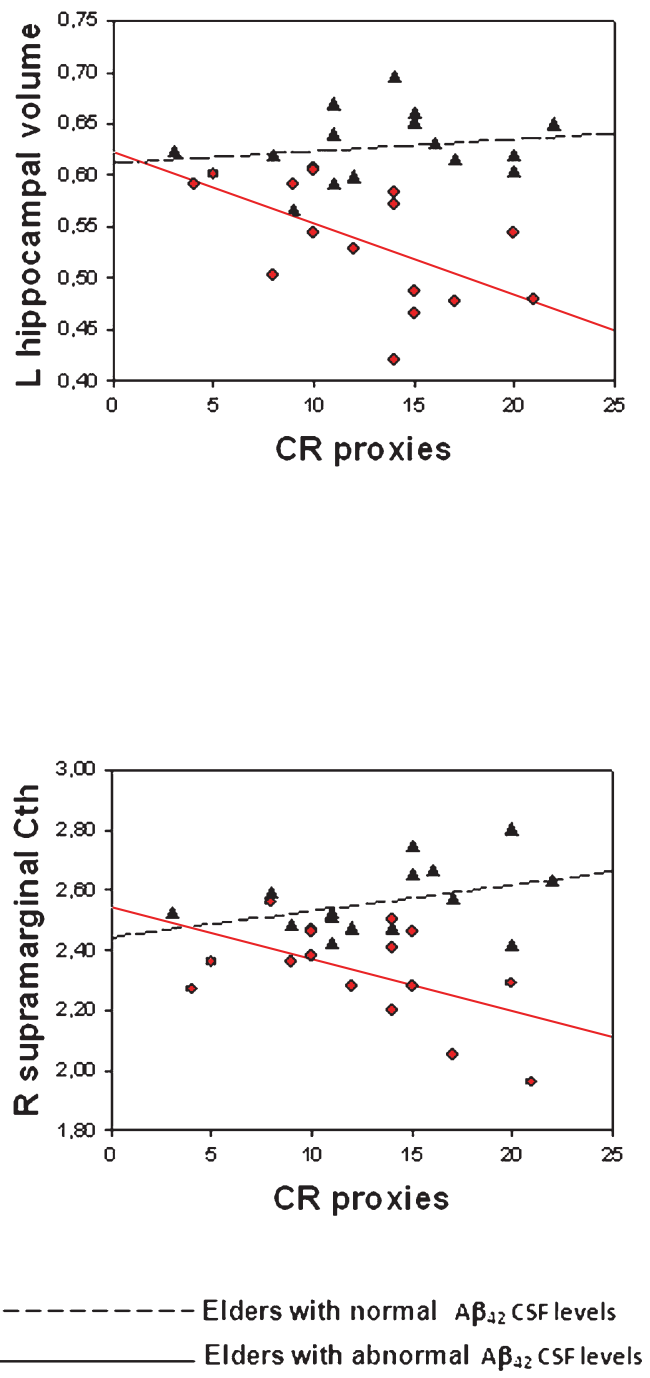

Fig. 2. Scatter plots showing interactions between cognitive reserve proxies and $A \beta_{42}$-related areas of cortical thinning or atrophy in elderly with normal versus abnormal $A \beta_{42}$ CSF levels. In red, statistically significant correlation coefficients (see Results for statistical details). Cth: cortical thickness; CR: cognitive reserve; R: right; L: left.

deposition (PiB-PET imaging or CSF levels), differences in the criteria used to dichotomize the sample, and the differential treatment of potential nuisance variables vary across studies and may lead to differences. For example, in the present study we were specifically interested in the relationships between $\mathrm{A} \beta_{42}$ and cognitive reserve proxies (see Introduction), and so we introduced $A P O E$ status and p-tau levels as covariates in order to isolate $A \beta_{42}$-related brain changes. Finally, the mean age of our subjects with abnormal $A \beta_{42}$ levels was lower than in the rest of the studies. This may partially explain why we did not find differences in the areas that have been described as having an important role in the preclinical phase of $\mathrm{AD}$ [49] and showing $A \beta_{42}$ related cortical thinning in cognitively healthy elderly $[46,48]$ such as the posterior cingulate or precuneus. Along these lines, a previous study [46] found that 
these regions showed age-related cortical thickness reductions. When the between-group comparison was carried out without controlling for age, gender, p-tau levels, and $A P O E$ status, precuneus and parahippocampal regions also showed $A \beta_{42}$-related cortical thinning (data not shown). Overall, our cortical thickness results lend support to earlier PiB and CSF studies suggesting that $A \beta_{42}$ deposition in normal elderly may represent the preclinical or asymptomatic AD phase.

When VBM analyses were carried out in a whole brain manner, we found a significant difference in the subjects with abnormal $A \beta_{42} \mathrm{CSF}$ levels compared to those with normal $A \beta_{42}$ CSF levels circumscribed to the left hippocampus. The finding of $A \beta_{42}$-related hippocampal atrophy in this study is in agreement with previous studies in cognitively healthy elderly harboring high $\mathrm{A} \beta_{42}$ deposition $[10,48,50]$, although conflicting results have also been reported [51]. Mormino et al. [50] studied the relationship between hippocampal volume, episodic memory, and $\mathrm{A} \beta_{42}$ deposition (as measured by $\mathrm{PiB}$ imaging) in three independent samples of cognitively healthy elderly. They found that higher $A \beta_{42}$ deposition was associated with smaller hippocampal volume across the three samples. Storand et al. [48] observed smaller hippocampal volumes in 29 participants with high $\mathrm{A} \beta_{42}$ deposition (measured by PIB-PET imaging). Finally comparing high and low $\mathrm{PiB}$ retention in cognitively normal subjects, Jack et al. [10] found that the hippocampus on average was more atrophic (though not significantly so) in the high PiB subgroup. With regard to the asymmetry shown in the present study (only the left hippocampus showed $A \beta_{42}$-related atrophy), previous investigations have consistently reported an asymmetry (left less than right) between the left and the right hippocampal volumes in healthy subjects, MCI, and AD [52]; the left hippocampus thus showed greater atrophy.

The differences in the results using VBM versus cortical thickness methods may appear surprising. However, previous studies also presented discrepancies [53, 54] due, perhaps, to differences in the sensitivity of the two measures. Cortical thickness is thought to be a more sensitive measure, because gray matter volume (measured by VBM) is dependent on the local cortical surface (and hence cortical folding) as well as on the local cortical thickness [55]. Based on previous evidence of neurodegenerative and psychiatric diseases using the same combination of methods $[56,57]$, we concluded that the combination of the two methods may provide complementary information. It is important to note that when a less strict statistical threshold was used for the VBM analyses (uncorrected $p<0.001$, data not shown) more extended regions of the temporal lobe and the frontal lobe showed greater atrophy in subjects with abnormal $\mathrm{A} \beta_{42} \mathrm{CSF}$ levels.

The main finding of our study was that there was a group-by-cognitive reserve proxy interactional effect on the left hippocampus and right supramarginal gyrus. Follow-up analyses showed that cognitive reserve proxies were significantly and negatively associated with hippocampal volumes and supramarginal cortical thinning in subjects with abnormal $A \beta_{42} \mathrm{CSF}$ levels but not in those with normal $A \beta_{42} \mathrm{CSF}$ levels. As groups did not differ on cognitive tests, the most straightforward interpretation within the theoretical framework of the reserve hypothesis is that elderly subjects with abnormal $A \beta_{42}$ CSF levels and with high cognitive reserve proxies exhibit more advanced gray matter atrophy while remaining cognitively normal. Although there were no significant between-group differences in task performance, memory performance showed a trend toward significance $(p=0.07)$. Some studies $[13,18,50]$, but not all [10], found $\mathrm{A} \beta_{42}$ deposition to be related to episodic memory performance. We therefore repeated the GLM (see Methods), controlling for memory performance. The interactional effect (group-by-cognitive reserve proxy) remained significant (data not shown). This suggests that the interactional effect is independent of interindividual differences in memory performance. Therefore, in accordance with the cognitive reserve theory, and taking $A \beta_{42}$ CSF levels as a surrogate for pathology, our results suggest that at a comparable level of cognitive performance, the underlying neuropathological process is more advanced in patients with higher cognitive reserve proxies [51].

Inverse relationships between cognitive reserve proxies and brain measures have been consistently found in AD studies [25]. This is corroborated by the results of the present study if we consider that our subjects with abnormal $A \beta_{42}$ CSF levels may in part represent the preclinical phase of AD. Nevertheless, previous studies in healthy elderly found that mental activity across the lifespan correlated with a reduced rate of hippocampal atrophy [58] or that healthy elderly subjects with higher education presented reduced mean diffusivity (indicating higher integrity) in the bilateral hippocampus [59]. Following these investigations, we might have expected to find a positive correlation reflecting more preserved hippocampal structure as a function of cognitive reserve in elderly with normal $\mathrm{A} \beta_{42}$ CSF levels. However, we did not record this observation, possibly due to a lack of statistical 
power caused by the relatively reduced sample size. It should also be borne in mind that the aim of this study was to investigate the differential relationships between cognitive reserve proxies and brain structure in $A \beta_{42}$-related areas within the two groups; therefore, we carried out analyses only in areas showing $\mathrm{A} \beta_{42}$-related atrophy. Hence, we cannot exclude the possibility that a group-by-cognitive reserve proxy effect or a relationship between cognitive reserve proxies and brain structure may exist in other brain regions that were not targeted in the present study. Actually, we tested this possibility in subsequent analyses and results tended to identify other areas besides the ones previously described (parahippocampus, inferior and superior temporal lobe in VBM analyses, and precentral gyrus in cortical thickness analysis). However, these results did not survive multiple comparison correction (data not shown). Thus, further studies are needed to clarify this issue.

The implications that these results may have for diagnosis or prognosis are based on previous evidence. On the one hand, an existing longitudinal study on subjects cognitively normal at base line [60] showed that those converting to dementia had significantly fewer years of education (a proxy of cognitive reserve) than non-converters; both education and CSF levels were predictors of conversion. On the other hand, the relevance of hippocampal volume to the conversion from $\mathrm{MCI}$ to $\mathrm{AD}$ has been stressed by several investigators [61-63]. Furthermore, we found greater cortical thinning in the supramarginal gyrus in elderly subjects with abnormal $A \beta_{42}$ CSF levels and higher cognitive reserve proxies; this area has been shown to present gray matter loss in patients with MCI. Moreover, longitudinal studies investigating the conversion to $\mathrm{AD}$ showed gray matter loss in this region (for a review, see [64]). Finally, a follow-up study comparing MCI patients either converting to dementia or remaining stable [22] reported that MCI converters with higher education had significantly lower $A \beta_{42}$ levels than those with lower education. This suggests that the accumulation of plaques was more pronounced in the highly educated group.

This study has several limitations. The first is the relatively small number of subjects included, which may have lessened the statistical power. However, the differences between elderly patients with normal and abnormal $A \beta_{42}$ CSF levels were observed after correcting for multiple comparisons (FWE corrected) and adjusting for the main confounding variables (age, gender, $A P O E$, and p-tau status). Moreover, the crosssectional design of the study does not allow us to test the hypothesis that elderly with abnormal $\mathrm{A} \beta_{42} \mathrm{CSF}$ levels and higher proxies of cognitive reserve may be at later stages of the AD preclinical phase (and will thus convert faster to MCI or AD). Solely longitudinal studies providing information about conversion to MCI or AD could properly test this hypothesis. Although functional resources might compensate for greater hippocampal atrophy in elderly with abnormal $\mathrm{A} \beta_{42} \mathrm{CSF}$ levels and higher cognitive reserve proxies, our study does not provide direct evidence of the implementation of cognitive reserve mechanisms in brain structure or brain functionality. Probably, functional imaging studies may help to clarify the mechanisms, underlying the fact that despite greater hippocampal atrophy, elderly with abnormal $A \beta_{42}$ CSF levels and high cognitive reserve proxies can perform at a similar cognitive level. Also, the group by cognitive reserve proxy interaction was not highly significant (see Results), thus further studies in larger samples are needed in order to confirm this finding. On a different subject, a strength of our study is that we used a comprehensive measure of cognitive reserve, including not only years of education but also cognitively stimulating activities. We also included subjects with and without memory complaints (see Methods). It has been suggested [18] that brain $A \beta_{42}$ burden is not related to brain atrophy in healthy subjects without memory complaints but only in subjects with memory complaints, thus the inclusion of subjects with memory complaints may have influenced our results. This issue needs to be studied in future investigations.

In summary, we provide evidence that in elderly people with abnormal $A \beta_{42}$ CSF levels, those with greater cognitive reserve proxies can tolerate more atrophy while remaining cognitively normal. Based on our findings, we hypothesize that elderly with abnormal $\mathrm{A} \beta_{42}$ CSF levels with higher cognitive reserve proxies are expressing higher neural compensation [16] (i.e., tolerating greater hippocampal atrophy) than elderly with abnormal $A \beta_{42}$ CSF levels with lower cognitive reserve proxies. These observations imply that cognitive reserve is significant even in cognitively preserved elderly adults that may represent in part the preclinical phase of $\mathrm{AD}$, that is, prior to clinical symptoms. At the clinical level, these results emphasize the relevance of evaluating cognitive reserve proxies in combination with neuroimaging techniques for early diagnosis. Finally, we demonstrate that $A \beta_{42}$ status could have been a confounding factor in earlier studies combining cognitive reserve with neuroimaging measures in samples of healthy elderly defined using clinical and neuropsychological information. 


\section{ACKNOWLEDGMENTS}

Eider M. Arenaza-Urquijo is supported by a Catalan Government predoctoral research fellowship. Dr. Bartrés-Faz is supported by a Spanish Ministerio de Ciencia e Innovación (SAF2009-07489) and by the Ministerio de Sanidad, Política Social e Igualdad (IMSERSO 200/2011) research grants. Dr. Molinuevo has provided scientific advice and has been an investigator or data monitoring board member receiving consultancy fees from Pfizer-Eisai, Janssen-Cilag, Novartis, Lundbeck, Roche, Bayer, Bristol-Myers Squibb, GE Health Care, GlaxoSmithKline, Merz, MSD and Innogenetics. Dr. Lorena Rami is supported by Servet grant as a senior investigator from the Spanish Ministry of Science (CP08/00147), FIS PI11/01071, Fondo europeo de desarrollo regional, una manera de hacer Europa, grant from IMSERSO 197/2011.

Authors' disclosures available online (http://www.jalz.com/disclosures/view.php?id=1682).

\section{REFERENCES}

[1] Jack CR Jr., Wiste HJ, Vemuri P, Weigand SD, Senjem ML, Zeng G, Bernstein MA, Gunter JL, Pankratz VS, Aisen PS, Weiner MW, Petersen RC, Shaw LM, Trojanowski JQ, Knopman DS (2010) Brain beta-amyloid measures and magnetic resonance imaging atrophy both predict time-to-progression from mild cognitive impairment to Alzheimer's disease. Brain 133, 3336-3348.

[2] Jack CR Jr., Vemuri P, Wiste HJ, Weigand SD, Aisen PS, Trojanowski JQ, Shaw LM, Bernstein MA, Petersen RC, Weiner MW, Knopman DS (2011) Evidence for ordering of Alzheimer disease biomarkers. Arch Neurol 68, 1526-1535.

[3] Sperling RA, Aisen PS, Beckett LA, Bennett DA, Craft S, Fagan AM, Iwatsubo T, Jack CR Jr., Kaye J, Montine TJ, Park DC, Reiman EM, Rowe CC, Siemers E, Stern Y, Yaffe K, Carrillo MC, Thies B, Morrison-Bogorad M, Wagster MV, Phelps CH (2011) Toward defining the preclinical stages of Alzheimer's disease: Recommendations from the National Institute on Aging-Alzheimer's Association workgroups on diagnostic guidelines for Alzheimer's disease. Alzheimers Dement 7, 280-292.

[4] Bennett DA, Schneider JA, Tang Y, Arnold SE, Wilson RS (2006) The effect of social networks on the relation between Alzheimer's disease pathology and level of cognitive function in old people: A longitudinal cohort study. Lancet Neurol 5, 406-412.

[5] Burns A, Tomlinson BE, Mann DM (1997) Observations on the brains of demented old people. B.E. Tomlinson, G. Blessed and M. Roth, Journal of the Neurological Sciences (1970) 11, 205-242 and Observations on the brains of non-demented old people. B.E. Tomlinson, G. Blessed and M. Roth, Journal of Neurological Sciences (1968) 7, 331-356. Int J Geriatr Psychiatry 12, 785-790.

[6] Davis DG, Schmitt FA, Wekstein DR, Markesbery WR (1999) Alzheimer neuropathologic alterations in aged cognitively normal subjects. J Neuropathol Exp Neurol 58, 376-388.
[7] Hulette CM, Welsh-Bohmer KA, Murray MG, Saunders AM, Mash DC, McIntyre LM (1998) Neuropathological and neuropsychological changes in "normal" aging: Evidence for preclinical Alzheimer disease in cognitively normal individuals. J Neuropathol Exp Neurol 57, 1168-1174.

[8] Price JL, Morris JC (1999) Tangles and plaques in nondemented aging and "preclinical" Alzheimer's disease. Ann Neurol 45, 358-368.

[9] Fotenos AF, Mintun MA, Snyder AZ, Morris JC, Buckner RL (2008) Brain volume decline in aging: Evidence for a relation between socioeconomic status, preclinical Alzheimer disease, and reserve. Arch Neurol 65, 113-120.

[10] Jack CR Jr, Lowe VJ, Senjem ML, Weigand SD, Kemp BJ, Shiung MM, Knopman DS, Boeve BF, Klunk WE, Mathis CA, Petersen RC (2008) 11C PiB and structural MRI provide complementary information in imaging of Alzheimer's disease and amnestic mild cognitive impairment. Brain 131, 665-680.

[11] Mintun MA, Larossa GN, Sheline YI, Dence CS, Lee SY, Mach RH, Klunk WE, Mathis CA, DeKosky ST, Morris JC (2006) [11C]PIB in a nondemented population: Potential antecedent marker of Alzheimer disease. Neurology 67, 446-452.

[12] Montine TJ, Peskind ER, Quinn JF, Wilson AM, Montine KS, Galasko D (2011) Increased cerebrospinal fluid F2isoprostanes are associated with aging and latent Alzheimer's disease as identified by biomarkers. Neuromolecular Med 13, 37-43.

[13] Pike KE, Savage G, Villemagne VL, Ng S, Moss SA, Maruff P, Mathis CA, Klunk WE, Masters CL, Rowe CC (2007) Beta-amyloid imaging and memory in non-demented individuals: Evidence for preclinical Alzheimer's disease. Brain 130, 2837-2844.

[14] Rowe CC, Ellis KA, Rimajova M, Bourgeat P, Pike KE, Jones G, Fripp J, Tochon-Danguy H, Morandeau L, O'Keefe G, Price R, Raniga P, Robins P, Acosta O, Lenzo N, Szoeke C, Salvado O, Head R, Martins R, Masters CL, Ames D, Villemagne VL (2010) Amyloid imaging results from the Australian Imaging, Biomarkers and Lifestyle (AIBL) study of aging. Neurobiol Aging 31, 1275-1283.

[15] Katzman R (1993) Education and the prevalence of dementia and Alzheimer's disease. Neurology 43, 13-20.

[16] Stern Y (2009) Cognitive reserve. Neuropsychologia 47, 2015-2028.

[17] Wilson RS, Barnes LL, Aggarwal NT, Boyle PA, Hebert LE, Mendes de Leon CF, Evans DA (2010) Cognitive activity and the cognitive morbidity of Alzheimer disease. Neurology $\mathbf{7 5}$, 990-996.

[18] Chételat G, Villemagne VL, Pike KE, Baron J-C, Bourgeat P, Jones G, Faux NG, Ellis KA, Salvado O, Szoeke C, Martins RN, Ames D, Masters CL, Rowe CC (2010) Larger temporal volume in elderly with high versus low beta-amyloid deposition. Brain 133, 3349-3358.

[19] Erten-Lyons D, Woltjer RL, Dodge H, Nixon R, Vorobik R, Calvert JF, Leahy M, Montine T, Kaye J (2009) Factors associated with resistance to dementia despite high Alzheimer disease pathology. Neurology 72, 354-360.

[20] Rentz DM, Locascio JJ, Becker JA, Moran EK, Eng E, Buckner RL, Sperling RA, Johnson KA (2010) Cognition, reserve, and amyloid deposition in normal aging. Ann Neurol 67, 353364.

[21] Roe CM, Mintun MA, Ghoshal N, Williams MM, Grant EA, Marcus DS, Morris JC (2010) Alzheimer disease identification using amyloid imaging and reserve variables: Proof of concept. Neurology 75, 42-48. 
[22] Rolstad S, Nordlund A, Eckerström C, Gustavsson MH, Zetterberg H, Wallin A (2009) Biomarkers in relation to cognitive reserve in patients with mild cognitive impairmentproof of concept. Dement Geriatr Cogn Disord 27, 194-200.

[23] Landau SM, Marks SM, Mormino EC, Rabinovici GD, Oh H, O'Neil JP, Wilson RS, Jagust WJ (2012) Association of lifetime cognitive engagement and low $\beta$-amyloid deposition. Arch Neurol 69, 623-629.

[24] Liu Y, Julkunen V, Paajanen T, Westman E, Wahlund LO, Aitken A, Sobow T, Mecocci P, Tsolaki M, Vellas B, Muehlboeck S, Spenger C, Lovestone S, Simmons A, Soininen H (2012) Education increases reserve against Alzheimer's disease-evidence from structural MRI analysis. Neuroradiology 54, 929-938.

[25] Bartrés-Faz D, Arenaza-Urquijo EM (2011) Structural and functional imaging correlates of cognitive and brain reserve hypotheses in healthy and pathological aging. Brain Topogr 24, 340-357.

[26] Jagust WJ, Mormino EC (2011) Lifespan brain activity, $\beta$ amyloid, and Alzheimer's disease. Trends Cogn Sci (Regul Ed) 15, 520-526.

[27] Dickerson BC, Bakkour A, Salat DH, Feczko E, Pacheco J, Greve DN, Grodstein F, Wright CI, Blacker D, Rosas HD, Sperling RA, Atri A, Growdon JH, Hyman BT, Morris JC, Fischl B, Buckner RL (2009) The cortical signature of Alzheimer's disease: Regionally specific cortical thinning relates to symptom severity in very mild to mild AD dementia and is detectable in asymptomatic amyloid-positive individuals. Cereb Cortex 19, 497-510.

[28] Coffey CE, Saxton JA, Ratcliff G, Bryan RN, Lucke JF (1999) Relation of education to brain size in normal aging: Implications for the reserve hypothesis. Neurology 53, 189-196.

[29] Querbes O, Aubry F, Pariente J, Lotterie J-A, Démonet J-F, Duret V, Puel M, Berry I, Fort J-C, Celsis P (2009) Early diagnosis of Alzheimer's disease using cortical thickness: Impact of cognitive reserve. Brain 132, 2036-2047.

[30] Bartrés-Faz D, Solé-Padullés C, Junqué C, Rami L, Bosch B Bargalló N, Falcón C, Sánchez-Valle R, Molinuevo JL (2009) Interactions of cognitive reserve with regional brain anatomy and brain function during a working memory task in healthy elders. Biol Psychol 80, 256-259.

[31] Foubert-Samier A, Catheline G, Amieva H, Dilharreguy B, Helmer C, Allard M, Dartigues J-F (2012) Education, occupation, leisure activities, and brain reserve: A population-based study. Neurobiol Aging 33, 423.e15-25.

[32] Rami L, Gómez-Ansón B, Bosch B, Sánchez-Valle R, Monte GC, Villar A, Molinuevo JL (2007) Cortical brain metabolism as measured by proton spectroscopy is related to memory performance in patients with amnestic mild cognitive impairment and Alzheimer's disease. Dement Geriatr Cogn Disord 24 274-279.

[33] Reisberg H, Ferris SH, Sclan SG (1993) Empirical evaluation of the global deterioration scale for staging Alzheimer's disease. Am J Psychiatry 150, 680-682.

[34] Fagan AM, Mintun MA, Shah AR, Aldea P, Roe CM, Mach RH, Marcus D, Morris JC, Holtzman DM (2009) Cerebrospinal fluid tau and ptau(181) increase with cortical amyloid deposition in cognitively normal individuals: Implications for future clinical trials of Alzheimer's disease. $E M B O$ Mol Med 1, 371-380.

[35] Rami L, Valls-Pedret C, Bartrés-Faz D, Caprile C, SoléPadullés C, Castellvi M, Olives J, Bosch B, Molinuevo JL (2011) [Cognitive reserve questionnaire. Scores obtained in a healthy elderly population and in one with Alzheimer's disease]. Rev Neurol 52, 195-201.
[36] Sled JG, Zijdenbos AP, Evans AC (1998) A nonparametric method for automatic correction of intensity nonuniformity in MRI data. IEEE Trans Med Imaging 17, 87-97.

[37] Fischl B, Liu A, Dale AM (2001) Automated manifold surgery: Constructing geometrically accurate and topologically correct models of the human cerebral cortex. IEEE Trans Med Imaging 20, 70-80.

[38] Ségonne F, Pacheco J, Fischl B (2007) Geometrically accurate topology-correction of cortical surfaces using nonseparating loops. IEEE Trans Med Imaging 26, 518-529.

[39] Dale AM, Fischl B, Sereno MI (1999) Cortical surface-based analysis. I. Segmentation and surface reconstruction. Neuroimage 9, 179-194.

[40] Fischl B, Dale AM (2000) Measuring the thickness of the human cerebral cortex from magnetic resonance images. Proc Natl Acad Sci U S A 97, 11050-11055.

[41] Smith SM, Jenkinson M, Woolrich MW, Beckmann CF, Behrens TEJ, Johansen-Berg H, Bannister PR, De Luca M, Drobnjak I, Flitney DE, Niazy RK, Saunders J, Vickers J, Zhang Y, De Stefano N, Brady JM, Matthews PM (2004) Advances in functional and structural MR image analysis and implementation as FSL. Neuroimage 23(Suppl 1), S208S219.

[42] Smith SM(2002) Fast robust automated brain extraction. Hum Brain Mapp 17, 143-155.

[43] Zhang Y, Brady M, Smith S (2001) Segmentation of brain MR images through a hidden Markov random field model and the expectation-maximization algorithm. IEEE Trans Med Imaging 20, 45-57.

[44] Desikan RS, McEvoy LK, Thompson WK, Holland D, Roddey JC, Blennow K, Aisen PS, Brewer JB, Hyman BT, Dale AM (2011) Amyloid- $\beta$ associated volume loss occurs only in the presence of phospho-tau. Ann Neurol 70, 657-661.

[45] Desikan RS, McEvoy LK, Thompson WK, Holland D, Brewer JB, Aisen PS, Sperling RA, Dale AM (2012) Amyloid- $\beta-$ associated clinical decline occurs only in the presence of elevated P-tau. Arch Neurol 69, 709-713.

[46] Becker JA, Hedden T, Carmasin J, Maye J, Rentz DM, Putcha D, Fischl B, Greve DN, Marshall GA, Salloway S, Marks D, Buckner RL, Sperling RA, Johnson KA (2011) Amyloid- $\beta$ associated cortical thinning in clinically normal elderly. Ann Neurol 69, 1032-1042.

[47] Fortea J, Sala-Llonch R, Bartrés-Faz D, Lladó A, SoléPadullés C, Bosch B, Antonell A, Olives J, Sanchez-Valle R, Molinuevo JL, Rami L (2011) Cognitively preserved subjects with transitional cerebrospinal fluid $\beta$-amyloid $1-42$ values have thicker cortex in Alzheimer's disease vulnerable areas. Biol Psychiatry 70, 183-190.

[48] Storandt M, Mintun MA, Head D, Morris JC (2009) Cognitive decline and brain volume loss as signatures of cerebral amyloid-beta peptide deposition identified with Pittsburgh compound B: Cognitive decline associated with Abeta deposition. Arch Neurol 66, 1476-1481.

[49] Protas HD, Chen K, Langbaum JBS, Fleisher AS, Alexander GE, Lee W, Bandy D, de Leon MJ, Mosconi L, Buckley S, Truran-Sacrey D, Schuff N, Weiner MW, Caselli RJ, Reiman EM (2013) Posterior cingulate glucose metabolism, hippocampal glucose metabolism, and hippocampal volume in cognitively normal, late-middle-aged persons at 3 levels of genetic risk for Alzheimer disease. JAMA Neurol 70, 320-325.

[50] Mormino EC, Kluth JT, Madison CM, Rabinovici GD, Baker SL, Miller BL, Koeppe RA, Mathis CA, Weiner MW, Jagust WJ (2009) Episodic memory loss is related to hippocampalmediated beta-amyloid deposition in elderly subjects. Brain 132, 1310-1323. 
[51] Stern Y (2002) What is cognitive reserve? Theory and research application of the reserve concept. J Int Neuropsychol Soc 8, 448-460.

[52] Shi F, Liu B, Zhou Y, Yu C, Jiang T (2009) Hippocampal volume and asymmetry in mild cognitive impairment and Alzheimer's disease: Meta-analyses of MRI studies. Hippocampus 19, 1055-1064.

[53] Blankstein U, Chen JYW, Mincic AM, McGrath PA, Davis KD (2009) The complex minds of teenagers: Neuroanatomy of personality differs between sexes. Neuropsychologia 47, 599-603.

[54] Voets NL, Hough MG, Douaud G, Matthews PM, James A, Winmill L, Webster P, Smith S (2008) Evidence for abnormalities of cortical development in adolescent-onset schizophrenia. Neuroimage 43, 665-675.

[55] Hutton C, Draganski B, Ashburnerx J, Weiskopf N (2009) A comparison between voxel-based cortical thickness and voxel-based morphometry in normal aging. NeuroImage 48, 371-380.

[56] Pereira JB, Ibarretxe-Bilbao N, Marti M-J, Compta Y, Junqué C, Bargallo N, Tolosa E (2012) Assessment of cortical degeneration in patients with Parkinson's disease by voxel-based morphometry, cortical folding, and cortical thickness. Hum Brain Mapp 33, 2521-2534.

[57] Hyde KL, Samson F, Evans AC, Mottron L (2010) Neuroanatomical differences in brain areas implicated in perceptual and other core features of autism revealed by cortical thickness analysis and voxel-based morphometry. Hum Brain Mapp 31, 556-566.
[58] Valenzuela MJ, Sachdev P, Wen W, Chen X, Brodaty H (2008) Lifespan mental activity predicts diminished rate of hippocampal atrophy. PLOS ONE 3, e2598.

[59] Piras F, Cherubini A, Caltagirone C, Spalletta G (2011) Education mediates microstructural changes in bilateral hippocampus. Hum Brain Mapp 32, 282-289.

[60] Fagan AM, Roe CM, Xiong C, Mintun MA, Morris JC, Holtzman DM (2007) Cerebrospinal fluid tau/beta-amyloid(42) ratio as a prediction of cognitive decline in nondemented older adults. Arch Neurol 64, 343-349.

[61] Apostolova LG, Dutton RA, Dinov ID, Hayashi KM, Toga AW, Cummings JL, Thompson PM (2006) Conversion of mild cognitive impairment to Alzheimer disease predicted by hippocampal atrophy maps. Arch Neurol 63, 693-699.

[62] Devanand DP, Liu X, Tabert MH, Pradhaban G, Cuasay K, Bell K, De Leon MJ, Doty RL, Stern Y, Pelton GH (2008) Combining early markers strongly predicts conversion from mild cognitive impairment to Alzheimer's disease. Biol Psychiatry 64, 871-879.

[63] Tapiola T, Pennanen C, Tapiola M, Tervo S, Kivipelto M, Hänninen T, Pihlajamäki M, Laakso MP, Hallikainen M, Hämäläinen A, Vanhanen M, Helkala E-L, Vanninen R, Nissinen A, Rossi R, Frisoni GB, Soininen H (2008) MRI of hippocampus and entorhinal cortex in mild cognitive impairment: A follow-up study. Neurobiol Aging 29, 31-38.

[64] Jacobs HIL, Van Boxtel MPJ, Jolles J, Verhey FRJ, Uylings HBM (2012) Parietal cortex matters in Alzheimer's disease: An overview of structural, functional and metabolic findings. Neurosci Biobehav Rev 36, 297-309. 